\title{
Moderate to severe plaque psoriasis - treatment with drugs of the classic scheme
}

\author{
Participants: \\ DMarcelo Arnone \\ André Vicente Esteves de Carvalho' \\ (iD) Maria Denise Fonseca Takahashi' \\ (D) Wanderley M Bernardo ${ }^{2}$ \\ Contact: arnones@uol.com.br
}

Final version: July 7st, 2018

1. Brazilian Society of Urology, Rua Real Grandeza, 108 sala 101 - Botafogo, Rio de Janeiro - RJ, Brasil 2. Brazilian Medical Association, Rua São Carlos do Pinhal, 324 - Bela Vista, São Paulo - SP, Brasil

http://dx.doi.org/10.1590/1806-9282.65.4.530

The Guidelines Project, an initiative of the Brazilian Medical Association, aims to combine information from the medical field in order to standardize producers to assist the reasoning and decision-making of doctors.

The information provided through this project must be assessed and criticized by the physician responsible for the conduct that will be adopted, depending on the conditions and the clinical status of each patient.

\section{INTRODUCTION}

Pharmacotherapy for psoriasis includes conventional systemic drugs and biological immunomodulators. Conventional systemic drugs are considered the first-line treatment for moderate to severe psoriasis, including methotrexate (MTX), cyclosporine, retinoid, and phototherapy. The first systemic therapy used was MTX, which was approved by the US Food and Drug Administration (FDA) for the treatment of psoriasis in $1971^{1}$.

\section{METHODOLOGY}

A systematic review of the literature was performed, with the descriptors in accordance with PICO, with P corresponding to patients with moderate to severe plaque psoriasis, I to intervention with methotrexate (MTX), cyclosporine, retinoid, and psoralen -ultraviolet therapy, and $\mathrm{O}$ to the outcome of effectiveness and safety. A search was conducted in the Medline-PubMed database to answer the clinical questions. The criteria for exclusion were non-randomized studies, weak strength of evidence, studies not related to PICO, articles in languages other than Portuguese, English or Spanish, articles with no full text available. The search strategies defined for each clinical question were:

- Psoriasis AND (methotrexate OR MTX) AND random*

- Psoriasis AND (acitretin OR neotigason) AND random*

- Psoriasis AND (ciclosporin OR ciclosporine OR cyclosporine OR sandimmun OR CsA OR CyA)

- Psoriasis AND (methotrexate OR MTX OR acitretin OR neotigason OR ciclosporin OR ciclosporine OR cyclosporine OR sandimmun OR CsA OR CyA) AND (etanercept OR TNFR-Fc fusion protein OR Enbrel OR Receptors, Tumor Necrosis Factor OR infliximab OR monoclonal antibody OR monoclonal antibodies OR Remicade OR MAb cA2 OR 
adalimumab OR Humira OR ustekinumab OR Stelara OR immunobiological therapy) AND random*

The evidence retrieved was selected from the critical assessment using discriminative instruments (scores): JADAD and GRADE for randomized clinical trials and New Castle Ottawa scale for observational studies.

\section{RESULTS}

What is the effectiveness and what are the risks of methotrexate in the systemic treatment of psoriasis?

Patients ( $>18$ years $(\mathrm{N}=120)$ with a diagnosis of chronic plaque psoriasis for at least 6 months received methotrexate, subcutaneously, (17.5mg $1 \mathrm{x}$ week) or placebo injections over the first 16 weeks of the study (phase 1). When PASI was not reduced by $50 \%$ after 8 weeks, the patients started receiving $22.5 \mathrm{mg}$ methotrexate per week or a placebo. Between weeks 16 and 52 (phase 2), the patients who started out with methotrexate remained with the same dose. Unless they were receiving $17.5 \mathrm{mg}$ and in week $24 \mathrm{did}$ not reach PASI 75, in which case the dose was increased to $22.5 \mathrm{mg}$. Patients who had received $22.5 \mathrm{mg}$ and whose PASI 50 was not reached in week 24 were excluded from continuing with the treatment. In week 16 , a PASI 75 response had been obtained in 37 (41\%) patients of the methotrexate group compared with three (10\%) patients in the placebo group (RR 3.93 , 95\% CI 1: 31-11, 81; $\mathrm{p}=0.0026)$. The dose increase to $22.5 \mathrm{mg} /$ week in week 8 occurred in 28 (31\%) patients of the methotrexate group. A total of 25 (27\%) patients of those who received methotrexate had a sPGA score of "without injury" (0) or "almost without injury" (1) at 16 weeks compared to two (7\%) patients who received the placebo; $16(18 \%)$ versus no patient, respectively, presented a PASI90 response. Of the 22 patients who initially received the placebo, five (23\%) had their dose increased to $22.5 \mathrm{mg}$ /week at week 24 . Five (55\%) patients receiving methotrexate (methotrexate group) had their doses increased to $22.5 \mathrm{mg} /$ week in week 24 . The response rates increased with the continued treatment with methotrexate, at week 52. A PASI 90 response was observed in almost $28 \%$ of the patients of both methotrexate-methotrexate and methotrexate-placebo methotrexate groups, and a sPGA score of 0 or 1 was found in almost $40 \%$ of the patients in both groups ${ }^{2}(\mathrm{~A})$.

Participants older than 18 years, with moder- ate to severe psoriasis, with impairment of at least $10 \%$ of the body surface area (BSA) and PASI score $\geq 10$ were randomized into 3 groups: 108 patients received adalimumab (ADA), subcutaneous, $80 \mathrm{mg}$ at week 0 , followed by $40 \mathrm{mg}$ per week for the next 15 weeks; and 110 patients received methotrexate (MTX), orally, 7.5 to $25 \mathrm{mg}$ per week for 16 weeks. The comparison was made with 53 patients with the use of placebo. After 16 weeks of follow-up, 79.6\% of the patients treated with adalimumab achieved PASI 75 , compared with $35.5 \%$ of the patients treated with methotrexate ( $p<0.001$ vs. adalimumab) and $18.9 \%$ for the group treated with the placebo ( $<<0.001 \mathrm{vs.}$ adalimumab $-\mathrm{NNT}=2$ and $\mathrm{p}<0.05$ vs methotrexate - NNT=6). There was a statistically significant improvement in the full recovery of lesions (PASI 100) in patients treated with ADA (16.7\%) compared to patients treated with methotrexate $(7.3 \%)$ or the placebo (1.9\%). As to adverse events, $73.8 \%$ of the patients of the ADA group, $81.8 \%$ of the MTX group, and $79.2 \%$ of the placebo group had at least one adverse effect. However, there was no statistically significant difference between the groups for infections, moderate to severe adverse events, and adverse events related to the drugs. Adverse events that led to the interruption of the study were more frequent in the methotrexate group, mainly because of events related to liver dysfunction. There were no reports of tuberculosis or deaths during the study ${ }^{1.3}(\mathrm{~A})$.

Patients with plaque psoriasis with the involvement of over $20 \%$ of body surface area were evaluated and randomized for the use of methotrexate $(n=20)$ and treatment with traditional Chinese Medicine (TCM) $(\mathrm{n}=21)$. MTX was initially administered at a dose of 2.5 to $5 \mathrm{mg}$. If the laboratory parameters remained normal after a week, the dose was increased to $10 \mathrm{mg}$ per week, followed by further increases of $2.5 \mathrm{mg}$ per week (not exceeding 30mg/ week). There was a significant difference in PASI in relation to change of the baseline at 6 months in the two intervention groups, with a $73.9 \%$ improvement of the methotrexate group, 15.1\% of the MTC group, and $32 \%$ in the placebo group. The difference between the MTX and placebo groups was significant at 2,4 , and 6 months of evaluation $(p<0.01, p<0.001$, and $p<0.01$, respectively, NNT $=3$ at the 6 th month), with no significant difference between TCM and the placebo group. The methotrexate group also showed significant improvement in the Physician Global Assessment (PGA) and in the Psoriasis Disability Index 
(PDI). Adverse effects were reported in 65\% of MTX patients, $48 \%$ of TCM patients, and $30 \%$ of the placebo group. Nausea, vomiting and increased hepatic enzymes were the signs and symptoms most often reported in the MTX group ${ }^{4}(\mathrm{~A})$.

\section{RECOMMENDATIONS}

The treatment for moderate to severe psoriasis with methotrexate showed a significant reduction of PASI in relation to the baseline at 2,4 , and 6 months of evaluation when compared to the placebo, with the number needed to treat ranging from 3 to 6 patients. The most frequent adverse effects were nausea, vomiting, and altered hepatic enzymes.

\section{What is the effectiveness and what are the}

risks of acitretin in the systemic treatment of psoriasis?

Authors have evaluated patients with chronic plaque psoriasis involving $>10 \%$ of the body surface area (serious illness) divided into three groups of treatment with acitretin: $25 \mathrm{mg}, 35 \mathrm{mg}$, or $50 \mathrm{mg}$ per day. The treatment protocol was continued until the patient reached PASI 75 or for 12 weeks. Patients who reached PASI 75 before 12 weeks were followed-up until the end of the study. In the event of a severe worsening of the condition (an increase of over $50 \%$ of the PASI Score) after the beginning of the acitretin treatment, patients were removed from the study. Clinical improvement was observed in all groups on the PASI score from week 0 to week 12. There was a reduction in the overall average of the initial PASI score from 10.8 to 4.0 at week 12 . The percentage decline in average scores of PASI from week 0 to week 12 was $54 \%, 76 \%$, and $54 \%$ in the $25 \mathrm{mg}, 35 \mathrm{mg}$, and $50 \mathrm{mg}$ groups of acitretin/ day, respectively. The reduction in the scores of the $35 \mathrm{mg}$ \day group was statistically significant $(\mathrm{P}<0.05)$. A PASI 75 was obtained in $47 \%, 69 \%$, and $53 \%$ of the patients of the acitretin 25,35 , and 50 $\mathrm{mg} /$ day groups, respectively. The majority of the adverse events were mucocutaneous mild to moderate, and dose-dependent ${ }^{5}(\mathrm{~A})$.

In another study, patients with plaque psoriasis affecting $10-70 \%$ of body surface area were randomized to the following doses of acitretin: 8 patients receiving $10 \mathrm{mg}$ to $25 \mathrm{mg}$ per day, or 16 patients receiving $50 \mathrm{mg}$ to $75 \mathrm{mg}$ per day. Patients who received acitretin in doses of 50 to $75 \mathrm{mg}$ a day showed significant improvement $(\mathrm{p}<0.05)$. Adverse effects occurred more in patients who received a dose of acitretin of $25 \mathrm{mg} /$ day or more, but they were generally mild, and there was no need to interrupt the treatment ${ }^{6}(\mathrm{~A})$.

\section{RECOMMENDATIONS}

The use of acitretin at a dose of 35 to $75 \mathrm{mg}$ a day, in patients with plaque psoriasis, moderate to severe, showed significant improvement of psoriatic lesions. The most frequent adverse effects are: cheilitis, peeling of palmoplantar regions, and alopecia, but did not result in the interruption of the treatment during the period of 12 weeks.

\section{What is the effectiveness and what are}

the risks of cyclosporine in the systemic treatment of psoriasis?

Patients with moderate to severe plaque psoriasis, in remission after treatment with continued cyclosporine (CsA) for 8 to 16 weeks, were evaluated; 162 received $5 \mathrm{mg} / \mathrm{kg} /$ day CsA orally for 2 consecutive days at the weekend and 81 received a placebo, on the same dose regimen. There was no statistically significant difference in clinical success rates at 24 weeks between the comparison groups (66.9\% for the CSA group and $53.2 \%$ for the placebo group, $p=0.072$ ). The time until the first relapse was significantly higher in the group using CsA $(p=0.023)$. CsA was well tolerated, with no differences regarding renal function and arterial pressure between those who received CsA or the placebo ${ }^{7}(\mathrm{~A})$.

The study evaluated patients with severe plaque psoriasis with PASI $\geq 18$, resistant to topical treatment, with 2 weeks without systemic treatment and 1 week without topical treatment. The parameter used for effectiveness was the improvement of the PASI score by $75 \%$ or obtaining the absolute value of PASI $\leq 8$. The mean reduction of the PASI score at the end of the induction phase was $69 \%$ in the CsA $2.5 \mathrm{mg} / \mathrm{kg} /$ day group and $89 \%$ in the CsA $5 \mathrm{mg} / \mathrm{kg} /$ day group $(p=0.0001, N N T=5)$. Eighty-six percent of the patients reported some adverse effect during the period of treatment (for up to 21 months), most of them mild to moderate. The most frequent events were hypertension, hirsutism/hypertrichosis, headache, paresthesia, nausea, abdominal discomfort, influenza symptoms, fatigue, tremors, edema, and renal dysfunction. The serious adverse events reported were 
the development of malignant diseases in 8 patients, namely 4 cases of skin cancer, and 2 patients with myocardial infarction ${ }^{8}(\mathrm{~A})$.

A group of patients with plaque psoriasis, with PASI of at least 15 , received cyclosporine at $1.25 \mathrm{mg} /$ $\mathrm{kg} /$ day initially, with an increase to $2.5 \mathrm{mg} / \mathrm{kg} /$ day or $5 \mathrm{mg} / \mathrm{kg} /$ day when there is no $10 \%$ reduction of the PASI after 2 weeks or of $30 \%$ after 6 weeks. Another group for comparison received cyclosporine at $2.5 \mathrm{mg} / \mathrm{kg} /$ day, which was increased to $5 \mathrm{mg} / \mathrm{kg} /$ day when there was no $10 \%$ reduction of the PASI after 2 weeks or of $30 \%$ after 6 weeks. By the end of the treatment, after 12 weeks, $18 \%$ of patients with an initial dose of $1.25 \mathrm{mg} / \mathrm{kg} /$ day and $56 \%$ of patients with an initial dosage of $2.5 \mathrm{mg} / \mathrm{kg} /$ day showed a PASI response ${ }^{3} 75$. The most frequent adverse effects were gastrointestinal changes, common cold, and viral infection$^{9}(\mathrm{~B})$.

\section{RECOMMENDATIONS}

The treatment for moderate to severe plaque psoriasis is more effective the higher the dose, and the dose of $5 \mathrm{mg} / \mathrm{kg} /$ day had best responses. The most relevant severe adverse effects reported in long-term treatments (21 months) were malignant diseases and hypertension. For patients who have an adequate therapeutic response in up to 16 weeks, the maintenance treatment with cyclosporin administered during the weekends demonstrated to prolong, safely and effectively, the support of the therapeutic response.

Is there a difference in effectiveness and risk when comparing the treatment of psoriasis using drugs from the classic scheme (methotrexate, acitretin, and cyclosporine) and immunobiologics?

A study was conducted with moderate to severe psoriasis patients, with impairment of at least 10\% of the body surface area (BSA) and PASI score $\geq 10$. All patients had had plaque psoriasis for at least 1 year, and their lesions had been stable for at least 2 months. The period without other treatments was of 2 weeks for topical treatment and phototherapy, and 12 weeks for biological treatment. The patients were randomized into 3 groups: 108 patients received adalimumab (ADA), subcutaneous, $80 \mathrm{mg}$ at week 0 , followed by $40 \mathrm{mg}$ per week for the next 15 weeks; and 110 patients received methotrexate (MTX), orally, 7.5 to $25 \mathrm{mg}$ per week for 16 weeks. The comparison was made with 53 pa- tients with the use of placebo. After 16 weeks of follow-up, $79.6 \%$ of the patients treated with adalimumab achieved PASI 75, compared with $35.5 \%$ of the patients treated with methotrexate ( $p<0.001 \mathrm{vs.}$ adalimumab NNT=3) and $18.9 \%$ for the group treated with the placebo $(p<0.001 \mathrm{vs}$. adalimumab and $\mathrm{p}<0.05$ vs. methotrexate). There was a statistically significant improvement in the full recovery of lesions (PASI 100) in patients treated with ADA (16.7\%) compared to patients treated with methotrexate $(7.3 \%)$ or the placebo (1.9\%). As to adverse events, 73.8\% of the patients of the ADA group, $81.8 \%$ of the MTX group, and $79.2 \%$ of the placebo group had at least one adverse effect. However, there was no statistically significant difference between the groups for infections, moderate to severe adverse events, and adverse events related to the drugs. Adverse events that led to the interruption of the study were more frequent in the methotrexate group, mainly because of events related to hepatic changes. There were no reports of tuberculosis or deaths during the study ${ }^{1,3,11,12}(\mathrm{~A})$.

Authors selected 868 patients aged from 18 to 75 years, with a diagnosis of moderate to severe plaque psoriasis, with the involvement of more than $10 \%$ of body surface area and PASI $\geq 12$. Of these, 653 patients received infliximab $5 \mathrm{mg} / \mathrm{kg}$ at weeks $0,2,6,14$, and 22; and 215 patients received MTX 15mg weekly, with an increase in dose to $20 \mathrm{mg}$ weekly at the 6 th week of treatment when the response of the PASI was lower than $25 \%$. The improvement was significantly higher in patients treated with infliximab ( $78 \%$ vs. $42 \%, \mathrm{p}<0.001, \mathrm{NNT}=3$ ). The difference between the groups regarding the PASI 75 response was observed from the second week of treatment. The proportion of patients who achieved PASI 90 was significantly higher $(p<0.001)$ in patients from the infliximab group. Serious adverse events were reported more often in the infliximab group, and the most severe were serious infections (tuberculosis, opportunistic infections such as pneumocystis pneumonia, listeriosis, atypical mycobacterial diseases, histoplasmosis, salmonellosis, and serious viral infections) and infusion-related reactions ${ }^{12}(\mathrm{~A})$.

Sixty patients were included in a study; of these, 30 received etanercept $50 \mathrm{mg}$, twice a week and 30 others received acitretin $0.4 \mathrm{mg} / \mathrm{kg} /$ day. A total of $56.7 \%$ of the patients of the etanercept group and $26.7 \%$ of the acitretin group $(\mathrm{p}<0.005, \mathrm{NNT}=4)$ had a response of PASI75 ${ }^{13}(\mathrm{~A})$. 


\section{RECOMMENDATIONS}

Adalimumab is more effective and well tolerated in the treatment of moderate to severe plaque psoriasis when compared with the use of methotrexate. The use of infliximab is more effective but brings a higher risk of severe infections and infusion reactions. The use of etanercept is also more effective than acitretin. Thus, immunobiologics are more effective in the treatment of moderate to severe plaque psoriasis when compared with classic treatments. Immunobiologics, with the exception of infliximab, have proven to be safer and better tolerated when compared with the conventional therapies for the treatment of moderate to severe plaque psoriasis.

\section{REFERENCES}

1. Saurat JH, Stingl G, Dubertret L, Papp K, Langley RG, Ortonne JP, Unnebrink K, Kaul M, Camez A; CHAMPION Study Investigators. Efficacy and safety results from the randomized controlled comparative study of adalimumab vs. methotrexate vs. placebo in patients with psoriasis (CHAMPION). Br J Dermatol. 2008 Mar;158(3):558-66. PubMed PMID: 18047523

2. Warren Richard B, Ulrich Mrowietz, Ralph von Kiedrowski, Johannes Niesmann, Dagmar Wilsmann-Theis, Kamran Ghoreschi, Ina Zschocke, Thomas M Falk, Norbert BIVadorn-Schlicht, Kristian Reich. An intensified dosing schedule of subcutaneous methotrexate in patients with moderate to severe plaque-type psoriasis (METOP): a 52 week, multicentre, randomised, double-blind, placebo-controlled, phase 3 trial. Lancet. 2017 Feb 4;389(10068):528-537. PubMed: PMID: 28012564

3. Reich K, Signorovitch J, Ramakrishnan K, Yu AP, Wu EQ, Gupta SR, Bao Y, Mulani PM. Benefit-risk analysis of adalimumab versus methotrexate and placebo in the treatment of moderate to severe psoriasis: comparison of adverse event-free response days in the CHAMPION trial. I Am Acad Dermatol. 2010 Dec;63(6):1011-8. PubMed PMID: 20933301

4. Ho SG, Yeung CK, Chan HH. Methotrexate versus traditional Chinese medicine in psoriasis: a randomized, placebo-controlled trial to determine efficacy, safety and quality of life. Clin Exp Dermatol. 2010 Oct;35(7):71722. PubMed PMID: 19925489

5. Dogra S, Jain A, Kanwar AJ. Efficacy and safety of acitretin in three fixed doses of 25,35 and $50 \mathrm{mg}$ in adult patients with severe plaque type psoriasis: a randomized, double blind, parallel group, dose ranging study. Eur Acad Dermatol Venereol. 2013 Mar;27(3):e305-11.Pubmed PMID: 22816881

6. Gupta AK, Goldfarb MT, Ellis CN, Voorhees J). Side-effect profile of acitretin therapy in psoriasis. J Am Acad Dermatol. 1989 Jun;20(6):1088-93. PubMed PMID: 2526824

7. Colombo D, Cassano N, Altomare G, Giannetti A, Vena GA. Psoriasis relapse evaluation with week-end cyclosporine $A$ treatment: results of a randomized, double-blind, multicenter study. Int | Immunopathol Pharmacol. 2010 Oct-Dec;23(4):1143-52. PubMed PMID: 21244763.

8. Laburte C, Grossman R, Abi-Rached I, Abeywickrama KH, Dubertret L. Efficacy and safety of oral cyclosporin A (CyA; Sandimmun) for long- term treatment of chronic severe plaque psoriasis. Br | Dermatol. 1994 Mar;130(3):366-75. PubMed PMID: 8148280.

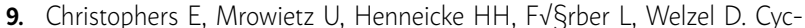
losporine in psoriasis: a multicenter dose-finding study in severe plaque psoriasis. The German Multicenter Study. I Am Acad Dermatol. 1992 Jan;26(1):86-90. PubMed PMID: 1732342.

10. Revicki D, Willian MK, Saurat JH, Papp KA Ortonne IP, Sexton C, Camez A. Impact of adalimumab treatment on health-related quality of life and other patient-reported outcomes: results from a 16-week randomized controlled trial in patients with moderate to severe plaque psoriasis. $\mathrm{Br}$ Dermatol. 2008 Mar;158(3):549-57. Epub 2007 Nov 28. PubMed PMID: 18047521.

11. Navarini AA, Poulin $Y$, Menter $A, G u Y$, Teixeira HD. Analysis of body regions and components of PASI scores during adalimumab or methotrexate treatment for patients with moderate-to-severe psoriasis. I Drugs Dermatol. 2014 May;13(5):554-62.

12. Barker J, Hoffmann M, Wozel G, Ortonne JP, Zheng H, van Hoogstraten $H$, Reich K. Efficacy and safety of infliximab vs. methotrexate in patients with moderate-to-severe plaque psoriasis: results of an open-label, active-controlled, randomized trial (RESTORE1). Br J Dermatol. 2011 Nov;165(5):1109-17. PubMed PMID: 21910713.

13. Caproni M, Antiga E, Melani L, Volpi W, Del Bianco E, Fabbri P. Serum levels of IL-17 and IL-22 are reduced by etanercept, but not by acitretin, in patients with psoriasis: a randomized-controlled trial. J Clin Immunol. 2009 Mar;29(2):210-4. PubMed PMID: 18763027.

14. Jadad AR, Moore RA, Carroll D, Jenkinson C, Reynolds DJ, Gavaghan DI, et al. Assessing the quality of reports of randomized clinical trials: is blinding necessary? Control Clin Trials 1996; 17:1-12.

15. Goldet G, Howick J. Understanding GRADE: an introduction. J Evid Based Med 2013; 6:50-4

16. Wells G, Shea B, O'Connell D, Robertson J, Peterson J, Welch V, et al. The Newcastle-Ottawa Scale (NOS) for assessing the quality of nonrandomised studies in meta-analyses. Disponível em: http://www.ohri.ca/ programs/clinical_epidemiology/oxford.asp.

17. Levels of Evidence and Grades of Recommendations - Oxford Centre for Evidence Based Medicine. Disponível em URL: http://cebm.jr2.ox.ac.uk/ docs/old levels.htm. 